\title{
MAD-4-MITO, a Multi Array of Detectors for ground-based mm/submm SZ observations
}

\author{
L. Lamagna*, M. De Petris*, F. Melchiorri*, E. Battistelli*, M. De Grazia*, G. \\ Luzzi* $^{*}$ A. Orlando* and G. Savini* \\ *Experimental Cosmology Group, Dipartimento di Fisica, Università di Roma "La Sapienza" \\ P.le A. Moro, 2 - 00185 ROMA (Italy)
}

\begin{abstract}
The last few years have seen a large development of $\mathrm{mm}$ technology and ultra-sensitive detectors devoted to microwave astronomy and astrophysics. The possibility to deal with large numbers of these detectors assembled into multi-pixel imaging systems has greatly improved the performance of microwave observations, even from ground-based stations, especially combining the power of multi-band detectors with their new imaging capabilities. Hereafter, we will present the development of a multi-pixel solution devoted to Sunyaev-Zel'dovich observations from ground-based telescopes, that is going to be operated from the Millimetre and Infrared Testagrigia Observatory.
\end{abstract}

\section{INTRODUCTION}

The Experimental Cosmology Group at the University of Rome "La Sapienza" has long been active in the development of instrumentation devoted to microwave astronomy from ground-based stations and balloon-borne platforms. In particular, observational campaigns have been performed from the Testa Grigia Observatory (3500m a.s.l.) searching for Sunyaev Zel'dovich effect on nearby clusters of galaxies, with special regard for the Coma cluster (A1656). Thanks to the experience accumulated in dealing with the site characteristics and the general issues of such measurements, and due to the importance of the SZ effect as a cosmological probe and a unique source of information on cluster physics, the instrument is going to be upgraded from a single-pixel, 4-band photometer into a multi-band bolometer array. It is planned to become operational by the end of 2002, when it will be employed to perform a systematic arcminute-scale search for the effect over a sample of nearby clusters.

\section{THE SUNYAEV ZEL'DOVICH EFFECT AS A PURE ESTIMATOR ON THE COSMOLOGICAL DISTANCE LADDER}

The full power of SZ measurements in providing systematic-free and redshift independent estimations of cosmological parameters has been widely explored in recent papers [1], as well as demonstrated from a series of experimental results from interferometric arrays and single dish observations in the radio region [2].

SZ effect [3] arises from inverse Compton scattering of CMB photons over a population of hot electrons present in galaxy clusters atmospheres, and is separated into a thermal component, due to the effect of velocity distribution in the hot gas, and a kinematic component, arising from the bulk motion of the hot gas along the line of sight. While the latter has not yet been object of a systematic search (apart from few ground-based observational campaigns from the SUZIE group), the thermal SZ effect has already been observed from a wide variety of instruments, mainly operating in the radio region with single dish or interferometric techniques. Combining SZ measurements over a cluster of galaxies with accurate $\mathrm{X}$-ray surface brightness information provided from satellite platforms yields an estimate of the cluster angular diameter distance, so that a sampling of the redshift-distance Hubble diagram is possible. This can bring out an estimate of the main cosmological parameters $\left(H_{0}\right.$ and $\left.\Omega_{m}\right)$ along with information on the total mass of the cluster. In particular, the possibility to extract the Hubble constant $H_{0}$ from a combination of SZ and X-ray data makes this 


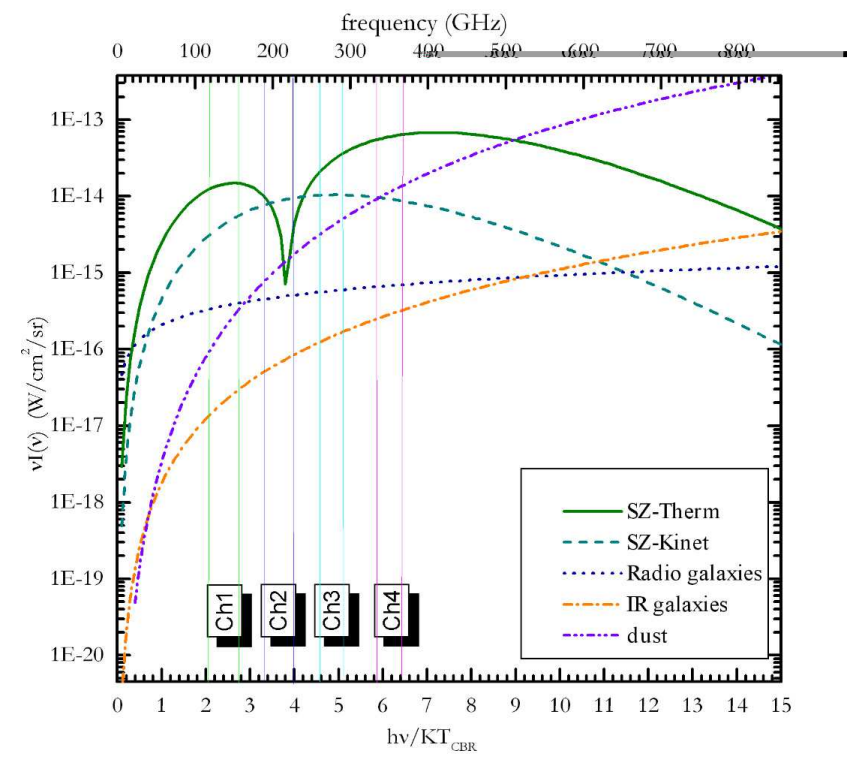

FIGURE 1. Main astrophysical contributions to signal in the 4 bands selected for the MAD experiment.

kind of measurements the natural complement to the mainly $\Omega$-sensitive CMB power spectrum information.

\section{SZ observation program from MITO}

The Millimetre and Infrared Testagrigia Observatory has been devoted to SZ search for the last 3 years, during which the main issues of SZ detection (at instrumental as well as at data reduction level) have been studied and cleared with the aid of a wide field $\left(\sim 17^{\prime}\right)$ single pixel photometer operating in 4 bands, chosen to match the main features of thermal SZ spectrum with the highest transparency windows of the atmospheric emission spectrum. The Coma cluster (A1656) has been used as a benchmark for testing instrument and observation strategies (drift-scans with azimuthal 3-field modulation have been performed recently), along with software simulation capabilities and data reduction techniques. The latter, in particular, are based on the combination of spectral and spatial cross-correlation of sky signals (which include both CMB data and atmospheric noise) in the spectral bands [4, 5].

\section{THE NEW MAD-4-MITO}

MAD (Multi Array of Detectors) is an experiment designed to operate in the 4 MITO bands (140,220,270 and $350 \mathrm{GHz}$ ). These have been chosen to match the best atmospheric transparency spectral windows and, at the same time, to exploit the full potential of thermal SZ effect spectral signature (see fig. 11). The highest frequency channel has been implemented to monitor the foreground contribution of galactic dust emission, which becomes more significant with increasing frequencies and decreasing galactic latitudes. Each channel is designed to receive radiation from the focal plane after free propagation in the optics system, and then it pixelizes the focal plane image into nine regions of $\sim$ 4' dimensions, arranged in a $9 \times 9$ bolometer array (see below). Thus, pixelization is performed only after splitting the focal plane into 4 identical spectrally defined beams, instead of employing optically insulated beam guides directly coupled to a single focal plane image. This ensures uniform band selection on each pixel, and should provide good efficiency to the whole system due to the few optical elements deployed between the telescope focal plane and each detector array. 


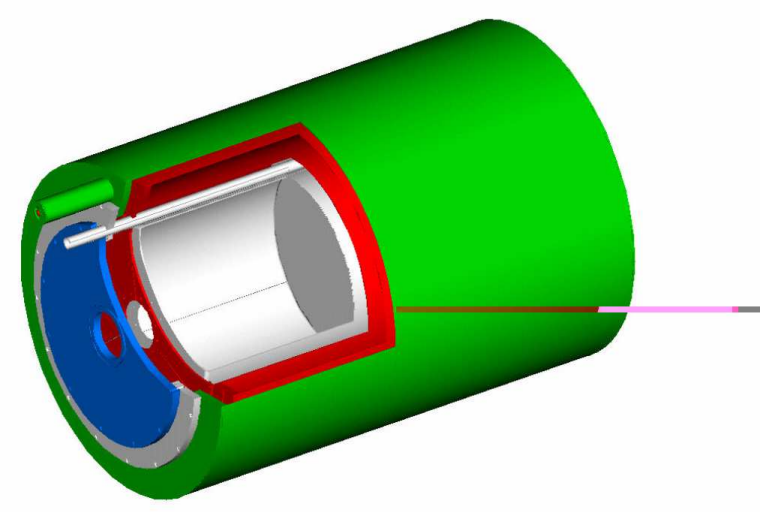

FIGURE 2. MAD cryostat layout. Short optical paths constrain the detector work area in the upper part of the dewar.

\section{Telescope}

MAD is designed to operate at the $2.6 \mathrm{~m}$ telescope of the Testa Grigia site, located at $3500 \mathrm{~m}$ a.s.1. on the Alps, just along the Italian/Swiss border line. The station, famous for the good atmospheric conditions and low water vapour content (less than 1mm during Winter months, with minimum peaks in December/January), ensures ideal observing conditions for microwave experiments, and has been used by the Rome group along the years as operating base for its main single-pixel SZ-devoted experiment [6], and as a benchmark site for the Diabolo cryostat [7]. The telescope has an aberration-free field of view of 20', which will be fully pixelized from the MAD optic system. Recent improvement in the alignment of the different optical components (especially the wobbling subreflector) and baffling at the focal plane level, ensure good control of modulated spurious signals, which may propagate through the demodulation system as slowly variable offsets, with time frequencies similar to those of the astrophysical signal coming from drift-scanned SZ sources, and thus potentially hard to discriminate on the basis of pure Fourier analysis.

\section{Cryostat}

As any other component of the experiment, MAD's cryostat has been designed to ensure long operation times with minimal servicing, enabling us to concentrate on observations and treatment of newly acquired data from calibration and SZ sources while still performing observations at the telescope.

The cryostat (fig. 2) is designed to have an upper radiation window, with the work area limited to a few tens of cm immediately below. This is necessary to assemble the minimum number of optical elements in front of the detectors and still have the possibility to operate in 4 different bands: the system is characterized from extremely small optical paths from the telescope focal plane to the four detector arrays (fig. 3 ) and thus needs to be concentrated in the upper part of the cryostat. This brought to the need of a good thermalization system, with the helium tank directly below the optics box, and the full height nitrogen tank providing $77 \mathrm{~K}$ radiation shielding by itself. The goal performance of the cryostat, whose assembling is still on its way, is to have a fully thermalized system at $4 K$ for $7-8$ days of continuous operation, without any need for cryogen refilling. With the heat inputs from radiation window, internal tank supports, and wiring for 40 detectors, it should need about $30 l$ for each of the liquid cryogens to reach this performance. Due to internal layout and tank shaping, the cryostat still remains very compact, with an external height of less than $70 \mathrm{~cm}$.

\section{Two-stage $\mathrm{He}^{3}-\mathrm{He}^{4}$ refrigerator}

Detector cool-down to the operating temperature of $300 \mathrm{mK}$ will be made possible by means of a two stage $H e^{3}-H e^{4}$ adsorption refrigerator. Each of the two parts of the system is made of a self-contained condensation 


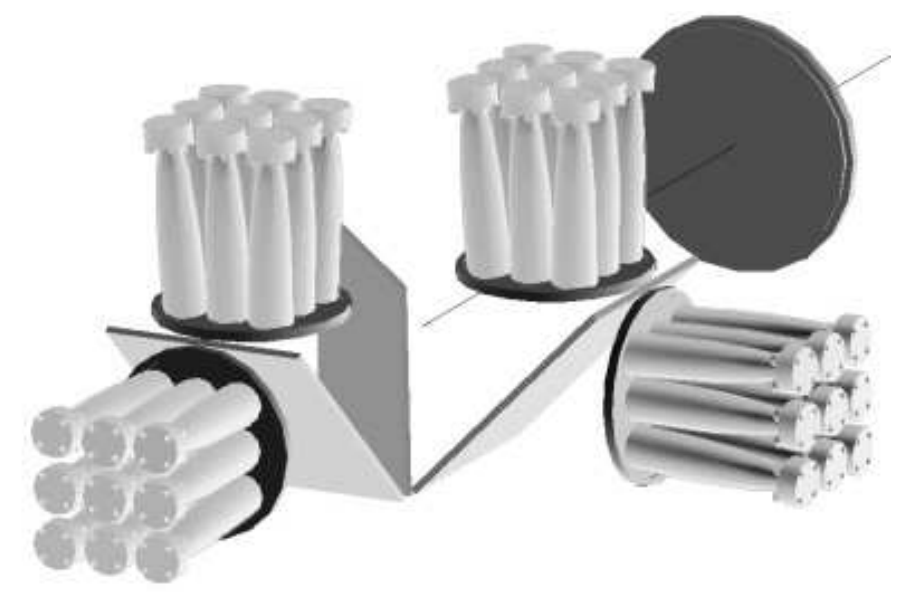

FIGURE 3. Sketch of detector layout in the MAD optics box. The circular shape indicates the position of cryostat radiation window

chamber, directly connected to the cold stage, a heat exchanger, thermally connected with the main $\mathrm{He}^{4}$ bath, and a cryopumping chamber filled with the proper amount of active charcoal, which may be thermally controlled using an externally powered heater and an electro-mechanical heat switch. The system uses cryopumping on $\mathrm{He}^{4}$ chamber to bring the cold stage below $\mathrm{He}^{3}$ condensation temperature, then the second cryopump lowers the liquid $\mathrm{He}^{3}$ (and thus the cold stage) temperature to the operating level of $\sim 300 \mathrm{mK}$. The same refrigerator has been successfully used with the present MITO cryostat, reaching $290 \mathrm{mK}$ limit temperature and more than 80 hours of continuous operation with a total heat input of $65 \mu \mathrm{W}$ on the cold stage [8]. One of the main advantages of this design is the total self-consistency of the fridge operation, since it doesn't need pumping on the main $\mathrm{He}^{4}$ bath with external devices.

\section{Detectors and Optics}

MAD's detectors, built at Haller-Beeman, Inc., are standard composite bolometers made of a sapphire radiation absorber and an NTD Ge thermistor. They are suspended within a heat trap, optimized for the 4 operating wavelengths, by means of 30 $\mu \mathrm{m}$ nylon fibers that keep the absorber firm at half-wavelength height and keep the whole system response fast (expected time constant, still to measure at this time, is $\tau \simeq 8 \div 12 \mathrm{~ms}$ ). Electrical responsivity is about $1.5 M V / W$.

Each of the 4 arrays is arranged in a $3 \times 3$ layout (fig. 3), with a tenth bolometer used as a blind monitor of system noise and channel cross-talk. Since the whole MITO telescope corrected focal plane area undergoes such pixelization, single pixel angular resolution is $\sim 4 .^{\prime}$. The spectral selection is performed over the unpixelized image, directly below the focal plane, and the beam is splitted into four images by means of low pass mesh filters operating at 45 incidence. Single bands are then selected from band-pass filters directly above the multi-mode Winston concentrators that couple detectors with the spectrally selected image. We think that this solution will ensure good optical efficiency to the system, especially if compared to closed light-pipe solutions, but we are aware of the problems that can arise from the need for large filters with uniform performance in order to have pixel-independent spectral selection. The focal plane layout, provided that the sky image is kept fixed on each array through the sidereal motion, allows for continuous detection over the desired direction and the nearest ones, and, above all, allows for simultaneous observation of the pixelized sky region in the different bands: this will ensure low systematics arising from time-delayed signal detection over the pixels and the different spectral regions.

\section{Readout electronics and data reduction}

Each detector is DC coupled with a bias circuit and a differential readout circuit, designed to keep low levels of microphonics and e.m. spurious signals. Low output impedance is achieved by bufferizing the two outputs of each detector through a dual JFET amplifier, set to a common drain (i.e. unity gain) configuration. Finally, the signal is 
amplified from a warm differential preamplifier board, which sends signals directly to data acquisition hardware. Proper filtering at lowest frequencies and anti-aliasing makes it possible to perform data acquisition at few tens of samples/second with 16 bit resolution. We will employ a commercial software controlled ADC board, for which fast monitoring software has been developed. Since the default observation strategy is based on signal modulation through wobbling of the telescope subreflector, on-the-fly demodulation has to be performed in order to extract the information content from each signal. This feature has been included in the acquisition software, together with specific selfcalibraton functions, such as offset-monitoring, phase shifting of the different signals with respect to the modulation reference, and others still to test on the field. We have also included the possibility of offline access to modulated data, since we plan to try many other different observation strategies that would allow for multi-mode demodulation over the same modulated signal, such as triangle wave azimuthal scanning or even total power unmodulated scanning, depending on the final detector performance and contingent weather conditions that could make it possible to remove the bulk of atmospheric noise by pixel cross-correlation alone.

\section{CONCLUSIONS}

We have shown the main characteristics of the MAD project, a multi-pixel 4-band detector designed for SZ search from ground-based stations, that will become operational from the Testa Grigia station within next Winter. The project headlines have been designed on the basis of the experience accumulated in the last 3 years with the single-pixel instrument that is still operational at the MITO telescope, and has already allowed for SZ detection over the Coma cluster. With the new instrument, we plan to perform an extensive observation program to measure the SZ effect over few tens of clusters and thus map the Hubble diagram from SZ detection over arcminute scales. This would also bring out many candidate SZ sources to include in the upcoming OLIMPO balloon-borne experiment observation program that is undergoing planning and design in our group.

\section{ACKNOWLEDGMENTS}

This project is being funded from MURST and University of Rome "La Sapienza". We also wish to thank P. de Bernardis and S. Masi for continuous support during our work.

\section{REFERENCES}

1. Rephaeli, Y., "The SZ effect: current status and future prospects", in 2K1BC Workshop - Experimental Cosmology at Millimetre Waves, edited by M. D. Petris and M. Gervasi, AIP Conference Proceedings, American Institute of Physics, New York, 2002.

2. J.E. Carlstrom, et al., "The Sunyaev-Zel'dovich Effect: Results and Future Prospects", in Constructing the Universe with Clusters of Galaxies, edited by F. Durret and G. Gerbal, IAP conference, 2001.

3. Sunyaev, R., and Zel'dovich, Y., Comm. Astrophys. Space Phys., 4, 173 (1972).

4. M. De Petris, et al., ApJ Letters (2002, in press).

5. L. Lamagna, et al., "SZ Effect Detection towards the Coma Cluster with the MITO experiment", in Proceedings of the Ninth Marcel Grossman Meeting, World Scientific, 2001.

6. M. De Petris, et al., New Astronomy, 1, 121 (1996).

7. A. Benoit, et al., Astron. Astrophys. Suppl. Ser., 141, 523-532 (2000).

8. T. Maiani, et al., Cryogenics, 39, 459 (1999). 